\title{
Analysis of a Very Large Web Search Engine Query Log
}

\author{
Craig Silverstein \\ Google Inc. \\ 2400 Bayshore, Mountain View, CA 94043 \\ csilvers@google.com* \\ Hannes Marais \\ Compaq Systems Research \\ 130 Lytton Ave, Palo Alto, CA 94301 \\ marais@pa.dec.com
}

\author{
Monika Henzinger \\ Google Inc. \\ 2400 Bayshore, Mountain View, CA 94043 \\ monika@google. com $^{\dagger}$ \\ Michael Moricz \\ Doublebill.Com, Inc. \\ 1800 Bridge Parkway, Redwood City, CA 94065 \\ michael.moricz@doublebill.com ${ }^{\ddagger}$
}

\begin{abstract}
In this paper we present an analysis of an AltaVista Search Engine query $\log$ consisting of approximately 1 billion entries for search requests over a period of six weeks. This represents almost 285 million user sessions, each an attempt to fill a single information need. We present an analysis of individual queries, query duplication, and query sessions. We also present results of a correlation analysis of the log entries, studying the interaction of terms within queries. Our data supports the conjecture that web users differ significantly from the user assumed in the standard information retrieval literature. Specifically, we show that web users type in short queries, mostly look at the first 10 results only, and seldom modify the query. This suggests that traditional information retrieval techniques may not work well for answering web search requests. The correlation analysis showed that the most highly correlated items are constituents of phrases. This result indicates it may be useful for search engines to consider search terms as parts of phrases even if the user did not explicitly specify them as such.
\end{abstract}

\section{Introduction}

There is a large interest in finding patterns in and computing statistics of search engine query logs. However there are very few results that study the query logs of commercial search engines. One that does, by Jansen et al. [Jansen et al., 1998], studies only 51,000 queries (taken from the query logs of Excite), which is a small percentage of the number of queries asked on search engines each day.

Our goal is to study a larger number of search requests, collected over the course of several weeks. Such a large data set has several advantages. One, because it covers a relatively large time range, it is less likely to be affected by ephemeral trends in querying (such as searches related to a new movie being released). For another, the extended time

\footnotetext{
* This work was done while visiting Compaq Systems Research.

${ }^{\dagger}$ This work was done while at Compaq Systems Research.

${ }^{\ddagger}$ This work was done while at the AltaVista company.
}

range allows us to identify individuals' patterns of use. Finally, by including all times of day as well as many days, we ensure that input from the entire world, and not just that portion which is awake during the period the log covers, is included in our results. ${ }^{1}$

Most of the questions about web use we choose to answer do not differ significantly from questions others have asked about query logs. We are interesting in counting statistics such as which queries are most common, what is the average number of words per query, how many queries are included in the average user session, and so on. In addition, however, we study correlations between query terms, and also among other field values. Thus, we seek to answer questions such as, "Do users from the U.S. ask disproportionately about basketball?" or, "Do people view particularly many result pages when querying about sex?" We believe this is a fertile area for research and present first steps in this direction.

The paper is structured as follows: Section 2 describes the AltaVista search environment and query log. Section 3 and 4 contain the analysis involving single items and the correlation analysis respectively. We conclude in Section 5 .

\section{The AltaVista Search Environment}

The AltaVista search environment consists of several components that are important for analyzing queries: the engine itself, which is accessible from the web page ww. altavista.com, and the query logs, which store information about what queries are made to the engine.

\subsection{The AltaVista Search Engine}

AltaVista is based on weighted boolean search. There are two major search modes: simple querying and advanced querying. A simple query consists of a collection of words, which are ORed together. A pair of operators allows for other boolean operations: -word instructs AltaVista to ignore documents containing word and is thus a NOT operator; +word instructs AltaVista to ignore documents not containing word and is thus an AND operator. The " operator is a proximity operator: words within double quotes must be adjacent in a document for the document to match the query. Quotes are therefore used to enclose phrases.

We use the term query term to denote a word or a quotation-mark-enclosed phrase.

\footnotetext{
${ }^{1}$ Our results, however, cover only the logs of the main AltaVista engine and not of mirror sites outside the U.S.
} 
An advanced query is more explicitly boolean. In advanced query mode, and, or, and not are interpreted as boolean operators rather than as search terms. Advanced queries may also include the boolean operator near, which is a relaxed form of the " operator: the words on either side of near must be close - but not necessarily adjacent - in a document for the document to match the query.

Both simple and advanced queries support web-specific operators. For instance, the query host: $x x \cdot y y \cdot z z$ returns all documents found on the machine xx.yy.zz. To simplify our analysis, we ignore these operators and treat the query as a four-word query containing the words host, $\mathbf{x x}$, yy, and zz.

The user can control aspects of the search in ways other than modifying the query. For instance, a pull-down menu allows the user to restrict result pages only to pages in a particular language. In the advanced search, an input box allows the user to restrict the results to pages last modified on a certain date, or within a range of dates.

After a query is entered and the various other restrictions processed, AltaVista returns a screen consisting of 10 URLs, with information about each URL such as the title. These URLs are ranked in order of relevance to the query, as determined by AltaVista's internal relevance function. The user may click on any URL to explore the associated web page. In addition, the user may click on navigation buttons to explore other screens of URLs. By clicking on " 3 ," for instance, the user would be taken to result screen 3 , which has the the 21st-30th most relevant URLs. Note this differs from some other search engines, where the user can jump to only the next 10 or previous 10 relevant URLs at any time.

\subsection{The Altavista Query Log}

The AltaVista query log has many components, only some of which concern us here. The query $\log$ is a text file consisting of a series of requests. A request may consist of a new query or a new result screen for a previously submitted query. Each request includes the foilowing fields:

- A timestamp indicating when the query was submitted. The timestamp is measured in milliseconds since 1 January 1970.

- A cookie, which can be used to say whether two queries come from the same user (this field is blank if the user has disabled cookies);

- The query terms, exactly as submitted;

- The bf result screen, that is the requested range of search results;

- Other user-specified modifiers, such as a restriction on the result pages' language or date of last modification;

- Submission information, such as whether the query is a simple or advanced query; and

- Submitter information, such as the browser the submitter is using and the IP address of the submitting host.

\subsection{Sessions}

A session is a series of queries by a single user made within a small range of time. A session is meant to capture a single user's attempt to fill a single information need. By focusing on a single user, we attempt to separate two information needs being asked at the same time; by limiting the time gaps in a session, we attempt to separate two information needs by the same user asked at different times.

The use of cookies is integral to identifying sessions, because in theory every user has a unique cookie. In actual use the situation is not as clear cut: different people using the same browser will share a cookie, and some users disallow cookies altogether. The former situation is not damaging for identifying sessions, since it is unlikely two separate users will be using the same browser simultaneously.

For those queries in which the user has disallowed cookies, we use the pair "domain IP/web browser used" as a substitute for the cookie. This is a poor substitute for cookies, particularly for large ISPs such as AOL, where tens of thousands of users can share a single IP address. Over $96 \%$ of all queries in our experiments have cookie information, however, so even a poor substitute discriminator of users is unlikely to bias the results. To ensure a lack of bias, we ran our experiments on two data sets: the full data set, and a data set restricted only to those queries with cookie information. The results in both cases were very similar, so we report only the experiments performed on the full data set.

In addition to identifying unique users, a good sessioning algorithm has to determine when a query starts a new information need. We use the heuristic that queries for a single information need come clustered in time, and then there is a gap before the user returns to the search engine. We used a cutoff of 5 minutes. This means that as a user is entering queries, as long as the latest query is submitted within five minutes of the previous query from that user, the new query is part of the same session. If the gap is more than 5 minutes, the new query starts a new session.

Since in reality a user may try to fill two information needs in one sitting, our session-identification heuristic almost certainly underestimates the true number of sessions.

\subsection{The Query Log Data Set}

Much of this paper is devoted to exploring the characteristics of the data set we studied; this introductory section is intended merely to give an introduction to the data.

The data set is summarized in Table 1. Already a few issues are apparent. For instance, fully $15 \%$ of all requests were empty requests, i.e. requests containing no query terms. Two thirds of these are cases were users of the AltaVista advance query service not understanding how to fill in the form. Of the non-empty requests, $32 \%$ consisted of a request for a new result screen, while $68 \%$ (the $575,244,993$ non-empty queries) consisted of a request for the first result screen of a new query.

The queries were collected over 43 days, from 2 August 1998 to 13 September 1998. They include all the queries submitted to the main (US) AltaVista search engine over this time period.

\subsection{Experimental Setup}

Our experiments were performed on a Compaq AlphaServer 4100 with four $465 \mathrm{MHz}$. processors and 4 gigabytes of main memory, running OSF V4.0b. All code was compiled using gcc 2.7.2 and compiled with the -06 optimization option. The experiments took about 22 hours of wall clock time to run. The duplicates analysis of Section 3.2, because of its large memory requirement, was done separately from the rest of the experiments; it took 18 additional hours of wall clock time to run. 
Table 1: Statistics summarizing the query log contents used in our experiments. Empty requests had no query terms. A request consists of either a new query or a new requested result screen. Exact-same-as-before requests had the same query and requested result page as the previous request. The total number of unique, non-empty queries gives the cardinality of the set consisting of all queries.

Total number of requests:

Total number of non-empty requests:

Total number of non-empty queries:

Total number of unique, non-empty queries:

Total number of sessions:

Total number of exact-same-as-before requests:
$993,208,159$
$843,445,731$
$575,244,993$
$153,645,050$
$285,474,117$
$41,922,802$

\section{First-order Analysis of Queries}

First-order analysis of queries consists of all analysis that involves counting only single items, such as the frequency of query terms or the number of times a query has more than 20 result screens requested for it. This is in contrast to second-order analysis, which requires counting pairs of items, such as the number of times the terms computer and free occur in the same query.

The first-order analysis we performed falls into two categories: analysis of individual queries, and analysis of how queries are modified throughout sessions.

\subsection{Analysis of Individual Queries}

One contrast that was noted early in the history of web search is that searches on the web tend to have many fewer search terms than searches in more traditional information retrieval contexts [Jansen et al., 1998]. Though the gap has narrowed over time, as we see in Table 2 the number of words per query is still small on average (2.35). The same average query length was found by [Jansen et al., 1998].

The number of operators used per query is also small, as is seen in Figure 3. One interesting note is that the maximum number of operators in a query is higher than the maximum number of terms found in a query. We attribute this to queries with a large number of dashes, which we interpret as operators in this context. Such a query could result from a user cutting text from another source and pasting it into the AltaVista search box.

\subsection{Analysis of Query Duplicates}

A different type of question concerning queries is how often an individual query is asked. We conjectured that a small set of queries is repeated many times over the course of a day. Indeed our data shows that the 25 most common queries form fully $1.5 \%$ of the total number of queries asked in a 43 day period, despite being only $0.00000016 \%$ of the unique queries. We give a listing of the 25 most frequent queries in Table 4. Note that uppercase and lowercase queries were not collapsed in the table.

One surprising result is the frequency of the term applet. Examination of the logs shows that almost all queries containing the term were submitted by a robot.

In Table 5 we show part of a histogram of query duplication; that is, what percent of queries are asked only once, what percent are asked twice, and so forth. Counts are over all 43 days that we studied. We classified two queries as being the same if they had the same words with the same capitalization. (AltaVista is case sensitive.) We ignored word order and operators. That fact that almost two-thirds of all queries are asked only once in a 6 week period indicates that information needs on the web are quite diverse, or at least are specified in diverse ways.

Determining query duplication is expensive because it requires storing each unique query. For our analysis, with over a hundred million unique queries, it is infeasible to store each query in memory. Instead, we stored a one word fingerprint of each query, which is merely a hash value. We then stored the small fingerprint in a hashtable. This allowed us to count the frequency of each query but would not let us later say which queries were the most common. Therefore, when a query was seen more than 1000 times, we stored a map from the query to the fingerprint. Since almost all queries are asked only once, this reduced the memory requirement significantly.

Each fingerprint is 32 bits, so even with a hundred million queries it is unlikely two queries will have the same fingerprint value. Nevertheless, we possibly undercount slightly the number of unique queries, and overcount slightly the frequency of some queries, because of fingerprint collisions.

\subsection{Analysis of Sessions}

One of the most striking observation about sessions is most of them are very short. Fully $63.7 \%$ of all sessions consist of only one request: meaning only one query was entered and only one result screen was examined. Our analysis, unfortunately, does not enable us to determine how many of these simple sessions are so short because the information need was fulfilled so easily, because the user gave up in despair after seeing a single screen of results, or because the user was unaware of the usefulness of modifying the query or requesting further result screens. Furthermore, we miss those cases where the user modified the query after the 5 minute session timeout had expired.

Tables 6 and 7 reinforce the idea that sessions are often simple. However, on occasion a single query is modified hundreds of thousands of times, or thousands of result screens are requested. These numbers - like the large count for the term applet in Section 3.2- are likely due to robots or other automatic search agents. We found that the average number of queries per session is 2.02 and the average screens per query is 1.39. Jansen et al. [Jansen et al., 1998] report the average number of queries per session of 2.8 , and average number of screens per query of 2.21 . We expect the difference is due to differing definitions of "session": Jansen et al. did not seem to "time out" a session, nor do they mention using a technique for distinguishing individual users from other agents, such as proxies, that have unique cookies. It is also possible that Excite users differ from AltaVista users, or that Excite's relatively short query log was not representative. 
Table 2: Statistics concerning the number of terms per query. Only distinct queries were used in the count; queries with many result screen requests were not upweighted. The mean and standard deviation are calculated only over queries with at least one term.

$\begin{array}{ll}0 \text { terms in query: } & 20.6 \% \\ 1 \text { term in query: } & 25.8 \% \\ 2 \text { terms in query: } & 26.0 \% \\ 3 \text { terms in query: } & 15.0 \% \\ >3 \text { terms in query: } & 12.6 \%\end{array}$

max terms in query: $\quad 393$

avg terms in query: $\quad 2.35$

stddev of terms in query: 1.74

Table 3: Statistics concerning the number of operators - +, -, and, or, not, and near - per query. Only distinct queries were used in the count; queries with many result screen requests were not upweighted.

$\begin{array}{lrlr}0 \text { operators in query: } & 79.6 \% & \text { max operators: } & 958 \\ 1 \text { operator in query: } & 9.7 \% & \text { avg operators: } & 0.41 \\ 2 \text { operators in query: } & 6.0 \% & \text { stddev of operators: } & 1.11 \\ 3 \text { operators in query: } & 2.6 \% & & \\ \text { > } 3 \text { operators in query: } & 2.1 \% & & \end{array}$

Table 4: The 25 most popular queries, and how often they were asked in the 43 day test period. Only distinct queries were used in the count; queries with many result screen requests were not upweighted. $\mathrm{p}^{* * * *}$ is a vulgarity.

\begin{tabular}{lr} 
Query & Frequency \\
\hline sex & 1551477 \\
applet & 1169031 \\
porno & 712790 \\
mp3 & 613902 \\
chat & 406014 \\
warez & 398953 \\
yahoo & 377025 \\
playboy & 356556 \\
xxx & 324923 \\
hotmail & 321267 \\
[non-ASCII query] & 263760 \\
pamela anderson & 256559 \\
p**** & 234037 \\
sexo & 226705 \\
porn & 212161 \\
nude & 190641 \\
lolita & 179629 \\
games & 166781 \\
spice girls & 162272 \\
beastiality & 152143 \\
animal sex & 150786 \\
SEX & 150699 \\
gay & 142761 \\
titanic & 140963 \\
bestiality & 136578 \\
\end{tabular}

Table 5: Statistics concerning how often distinct queries are asked. Only distinct queries were used in the count; queries with many result screen requests were not upweighted. Percents are of the 154 million unique queries.

$\begin{array}{lrlr}\text { Query occurs 1 time: } & 63.7 \% & \text { max query frequency: } & 1,551,477 \\ \text { Query occurs 2 times: } & 16.2 \% & \text { avg query frequency: } & 3.97 \\ \text { Query occurs 3 times: } & 6.5 \% & \text { stddev of query freq: } & 221.31\end{array}$


Table 6: Statistics concerning the characteristics of query modification in sessions.

$\begin{array}{lrlr}1 \text { query per session: } & 77.6 \% & \text { max queries per session: } & 172325 \\ 2 \text { queries per session: } & 13.5 \% & \text { avg queries per session: } & 2.02 \\ 3 \text { queries per session: } & 4.4 \% & \text { stddev of queries/session: } & 123.40\end{array}$

Table 7: Statistics concerning the characteristics of result screen requests in sessions.

$\begin{array}{lrlr}\text { 1 screen per query: } & 85.2 \% & \text { max screens per query: } & 78496 \\ \text { 2 screens per query: } & 7.5 \% & \text { (2nd most screens: } & 5108 \text { ) } \\ \text { 3 screens per query: } & 3.0 \% & \text { avg screens per query: } & 1.39 \\ \text { > 3 screens per query: } & 4.3 \% & \text { stddev of screens/query: } & 3.74\end{array}$

For those situations where a query is modified, we see in Table 8 that in about $12 \%$ of the cases the query is modified by either adding or deleting terms or operators. In this situation, it is likely the user is satisfied with how the information need is expressed, but the query is either too specific or not specific enough. Even when adding a word, a user may be trying to restrict the search space rather than broaden it, since often the word is added with a - operator, with the goal of removing non-relevant pages that share a common word.

In over half the cases, some query terms are deleted from the query and other terms added. In these cases it is likely the user is modifying the query not to change the scope of the query, but to restate the information need.

Over a third of the cases result in the a total change, where no word is shared between the two modifications. These may result in the information need being refined or even changed on the basis of information gleaned in the first search. In some cases, a total change may result from improperly identifying sessions. Unfortunately we don't know how many times this occurs.

\section{Second-order Analysis of Queries}

Second-order analysis of queries is analysis that requires joint counts, that is, counts for pairs of items in the same query. Unlike first-order analysis, which requires only linear space in the quantity being counted, second-order analysis requires quadratic space. Because of this, we limit our attention to query terms and fields for second-order analysis, and furthermore we limit ourselves to the 10,000 most common terms found in queries. (We include all field values, since there are only a small number). Because of these restrictions, the memory requirement does not grow with the size of the data set, and it is possible to do second-order analysis on large data.

Our second-order analysis consists of finding correlations between items, where an item is either a query term, such as "new york", or a field value, such as language=fr (which indicates the user specified only pages written in French should be returned). In addition to studying 2-way correlation, we devise a method for augmenting our correlation analysis to find 3- and 4-way correlations, while keeping the space use quadratic.

Since we limit our attention to the 10,000 most common terms, many queries become empty because they contain only rarer terms. We limit our attention only to queries with at least one term in the top 10,000 - for this data set, $313,454,867$ distinct queries.

\subsection{The Chi-squared Test for Correlation}

While in reality queries consist of items, for the purpose of discussing correlation we consider the dual formulation that items decide whether or not to be in queries. Intuitively, if one item is found in $n_{1}$ fraction of queries and another, unrelated item in $n_{2}$ fraction, we would expect them both to occur together in $n_{1} n_{2}$ fraction of queries. If the actual cooccurrence percent deviates significantly from this expected value, we must question the assumption that the two items are independent of one another.

This intuition is captured formally in the chi-squared statistic [Agresti, 1990]:

$$
\begin{aligned}
\chi^{2}(a, b)= & \frac{[E(a b)-O(a b)]^{2}}{E(a b)}+\frac{[E(\bar{a} b)-O(\bar{a} b)]^{2}}{E(\bar{a} b)}+ \\
& \frac{[E(a \bar{b})-O(a \bar{b})]^{2}}{E(a \bar{b})}+\frac{[E(\bar{a} \bar{b})-O(\bar{a} \bar{b})]^{2}}{E(\bar{a} \bar{b})}
\end{aligned}
$$

In this formula, $O(a b)$ is the number of queries in which items $a$ and $b$ co-occur. $E(a b)$ is the expected number of queries in which they co-occur, under the independence assumption. As mentioned above, $E(a b)=O(a) O(b) / n$, where $n$ is the total number of queries. $O(\bar{a})$ is the number of queries in which $a$ does not occur.

The $\chi^{2}$ value has a chi-squared distribution. (In particular, for boolean data it has a chi-squared distribution with one degree of freedom.) Looking up values for the chisquared distribution in a table, we see that only $5 \%$ of the time does the $\chi^{2}$ value exceed 3.84 if the variables are actually independent. Therefore, if we see $\chi^{2}>3.84$, we say that $a$ and $b$ are correlated at the $95 \%$ confidence level.

\subsection{The Correlation Coefficient}

While the chi-squared test can detect the presence of correlation, it cannot judge its strength. For instance, if two items are actually correlated, their $\chi^{2}$ value will grow as $n$, the size of the data, grows. The correlation coefficient, $\rho$, is typically used to measure the strength of correlation. It is defined as follows:

$$
\rho(a, b)=\frac{\left(\sum_{i}\left(A_{i}-\mu_{a}\right)\left(B_{i}-\mu_{b}\right)\right)}{\sqrt{\sigma_{a}^{2} \sigma_{b}^{2}}}
$$


Table 8: How queries are modified within a session.

$\begin{array}{lr}\text { Adding terms: } & 7.1 \% \\ \text { Deleting terms: } & 3.1 \% \\ \text { Modifying operators only: } & 1.4 \% \\ \text { Totally changing the query: } & 35.2 \% \\ \text { Otherwise modifying query terms: } & 53.2 \%\end{array}$

(Adding one term: $5.4 \%$ )

(Deleting one term: 2.1\%)

(operators everywhere: $0.4 \%$ ) where $\mu_{a}$ is the mean value of item $a$ (that is, $O(a) / n$ ), $\sigma_{a}^{2}$ is the variance of $a$, and $A_{i}$ is 1 if and only if query $i$ contains $a$. The denominator is a scaling factor that keeps $\rho$ between -1 and 1. For boolean data, $\rho$ will equal 0 if $A$ and $B$ are independent, 1 if they are perfectly correlated, and -1 if they are perfectly negatively correlated.

The correlation coefficient is important for data mining applications because there is often enough data to find weak but significant correlations. The correlation coefficient can help the analyst concentrate on those correlations that are strong enough to make an impact. For instance, suppose that once a year a user enters a query volcano baklava, but otherwise the two terms are independent. With enough data, the chi-squared test will (correctly) say there is a correlation, but the correlation coefficient will be low, indicating that the correlation is weak. An analyst would be wise not to change policy based on this correlation.

It is clear, then, that $\chi^{2}$ and $\rho$ are complementary measures, and one does not supersede the other. With "too little" data, $\chi^{2}$ will be low but $\rho$ can be high; with "too much" data, $\chi^{2}$ can be high even in cases where $\rho$ is near zero.

Despite the differing roles of $\chi^{2}$ and $\rho$, for boolean data such as we have for query log analysis, the two are related. In particular,

$$
\chi^{2}=n \times \rho^{2} .
$$

This relationship means that for boolean data, the strongest correlations are also the most likely to be true correlations rather than statistical anomalies. For mining query logs, this is an important fact, because with over 10,000 items there are more than 100 million statistical tests being done. Even at the $95 \%$ confidence level, there will likely be made millions of false judgements of correlation. Concentrating on items with correlation coefficient above 0.2 , however, the expected number of false judgements is less than one.

\subsection{Results for Two-way Correlation}

We calculated correlation between items, consisting of 10,000 terms and 662 field values. Field values are boolean items of the form field=value. For instance, domain=nl is an item in all queries emanating from the Netherlands. After throwing out trivial correlations, such as domain=nl being negatively correlated with domain $=k r$, we were left with 110 correlated pairs. Some of these are displayed in Table 9.

One interesting pattern found in the highly correlated pairs is that the most highly correlated items are constituents of phrases. This indicates there are many situations where users are not using the " operator to tell AltaVista that the separate words constitute a single term.

One phrase that contributes to three of the entries in Table 9 is "Buffy the Vampire Slayer," a TV show. While presumably most queries containing one of these words contained all three, buffy and slayer are more highly correlated than pairs containing vampire. This is because terms like buffy and slayer are unlikely to occur in queries in other contexts, making the correlation strong. However, there are likely many queries concerning vampires but not the TV show, making the correlation less strong in this case.

One of the most frequent of the highly correlated queries is between www and com. The fact that ww and com are highly correlated is hardly surprising; both often occur in a URL and neither is likely to be a common query term in other contexts. This is an extreme example of the general fact that text is naturally correlated.

The correlation between people from Korea querying and asking for result pages in Korean is hardly surprising. As mentioned before, the term applet is often queried by a robot, which explains the correlation between the word "applet" and requesting a date-restricted set of pages.

One interesting set of correlations concern "referred" users which are users sent to AltaVista by an AltaVista partner, rather than those who typed in the URL explicitly or came from a bookmark. These users are correlated highly with sessions with many modifications, perhaps indicating these users spend more time than most modifying their query. They are also particularly likely to "restart" their queries, meaning they enter a query with no terms in common with their previous query. This may indicate users sent to AltaVista to fill one information need get distracted and try to fill others as well. Another possible explanation for both data points is that cookie information does not get forwarded from all the referring sites, meaning we are misidentifying sessions in this case.

A final, unsurprising result is that the term the is correlated with long queries. It is likely that queries containing such a contentless word are natural language queries.

\subsection{Results for Three- and Four-way Correlation}

If a pair of items is correlated, then by definition all supersets of that pair are correlated as well. Therefore, to find correlations involving more than two items, it is necessary to find pairs of items that are uncorrelated. In the case of text this is difficult, since text is naturally correlated. Therefore, typical methods of finding large correlated sets have proven unsuccessful for mining queries in query logs.

In order to find these larger correlations, we implemented a heuristic that "phrasifies" highly correlated terms. In this case, we took the 1000 item pairs with the largest (positive) correlation coefficient, as determined from the analysis in the Section 4.3. For each pair, we constructed a new term consisting of that pair of items. A query contains the new term only if it contains both items in the pair. Effectively, we are pretending the correlation coefficient of the pair of items is 1 - meaning they only occur together - so the correlation of the pair with a third item is equal to the correlation of the pair with either of the items individually. In reality, since the correlation of the pair is less than 1, we approximate but underestimate the true correlation between the three items. 
Table 9: Some highly correlated pairs of items. The data set consists of about 10,000 items and 313,000,000 queries.

\begin{tabular}{ll|rr} 
Term A & Term B & Count together $(O(A B))$ & $\rho$ \\
\hline cindy & crawford & 118558 & 0.7098 \\
persian & kitty & 75716 & 0.6830 \\
pamela & anderson & 453467 & 0.6451 \\
visual & basic & 177971 & 0.6325 \\
www & http & 2355010 & 0.3862 \\
\hline buffy & slayer & 12340 & 0.3989 \\
slayer & vampire & 13640 & 0.3088 \\
buffy & vampire & 12986 & 0.2766 \\
\hline lang=ko & domain=kr & 1030416 & 0.7281 \\
date=restricted & applet & 1165565 & 0.7273 \\
referred=yes & sessmodlen=4+ & 76257842 & 0.6388 \\
referred=yes & sessmod=restart & 46359290 & 0.5482 \\
the & qwords=6+ & 2417838 & 0.1886 \\
\hline
\end{tabular}

Table 10: Some highly correlated items. By "phrasifying" highly correlated pairs - represented by "(word1, word2)," — we obtain sets of three and four highly correlated items. The data set consists of about 10,000 items and 244,000,000 queries.

\begin{tabular}{ll|rr} 
Term A & Term B & Count together $(O(A B))$ & $\rho$ \\
\hline (links, kitty) & (persian, adult) & 40375 & 0.9438 \\
(www, http) & (http, com) & 127783 & 0.9353 \\
(harvard, business) & review & 12554 & 0.9215 \\
(used, car) & (used, prices) & 24702 & 0.8988 \\
(bluemountain, com) & (www, bluemountain) & 36722 & 0.8871 \\
(anderson, lee) & (pamela, lee) & 105208 & 0.7375 \\
(ibm, video) & (highlander, newsgroups) & 5261 & 0.5037 \\
(persian, kitty) & (persian, links) & 45322 & 0.4687
\end{tabular}

By repeating the same analysis as was done in Section 4.3 with the 1000 new items, we can find triples of items that are correlated, or even sets of four if two pairs are correlated. The results are shown in Table 10. Most of the 3- and 4way correlations involve phrases with three and four words in them, such as "Harvard Business Review" and "Persian Kitty adult links." A connection such as "IBM video" and "highlander newsgroups" seems more obscure, and indeed the low frequency indicates it is probably the result of a small number of users repeatedly modifying a query with these four words as the base.

For multi-word phrases, the choice of word grouping can affect the correlation score. For instance, (persian, kitty, adult, links) has a fairly weak correlation when one of the pairs is (persian, kitty). This is because the phrase persian kitty is often asked alone, as a single two-word query; it therefore correlated relatively weakly with adult and links. On the other hand, the pairs (kitty, links) and (persian, adult) obviously each only occur when the full query persian kitty adult links is entered.

Similary, (www, bluemountain, com) is only correlated when considering the pairs in Table 10. The correlation between bluemountain and (www, com) is very low.

Given these observations, it makes sense to consider a triple of words to be correlated only if all 3 pairwise partitions of the words yield strong correlations.

\section{Conclusions and Further Research}

In this paper we presented an analysis of an AltaVista query $\log$ containing almost 1 billion entries. We confirmed the conjecture that an average web user differs significantly from the user model assumed by the information retrieval community. Surprisingly, for $85 \%$ of the queries only the first result screen is viewed, and $77 \%$ of the sessions contain only 1 query, i.e., the queries were not modified in these sessions.

In the correlation analysis we considered the queries of all users and found the strongest correlations resulted from short queries that were actually single-term phrase queries. For future work it might be interesting to restrict the correlation analysis to long queries, with for instance more than ten terms, in hopes of finding correlations between concepts rather than merely terms.

Our analysis did not distinguish between requests issued by humans and requests issued by robots. In some cases, robot-initiated queries in the AltaVista logs resulted in a number of seemingly bizarre results. It is impossible for us to judge how much automated search techniques skewed the results of this study. It would be interesting to repeat the analysis with the request from robots discarded, assuming a method could be found to distinguish requests by robots from requests by humans.

\section{References}

[Agresti, 1990] A. Agresti. Categorical Data Analysis. John Wiley \& Sons, New York, 1990.

[Jansen et al., 1998] Jansen, B.J., Spink, A., Bateman, J., and Saracevic, T.1998 Real Life Information Retrieval: A Study of User Queries on the Web, SIGIR FORUM, $32(1): 5-17$. 